\title{
Relative importance of female-specific and non-female-specific effects on variation in iron stores between women
}

\author{
John B. Whitfield, ${ }^{1,2}$ Susan Treloar, ${ }^{2}$ Gu Zhu, ${ }^{2}$ Lawrie W. Powell ${ }^{2}$ and Nicholas G. Martin ${ }^{2}$ \\ ${ }^{1}$ Department of Clinical Biochemistry, Royal Prince Alfred Hospital, Sydney, and ${ }^{2}$ Queensland Institute of Medical \\ Research, Brisbane, Australia
}

Received 22 May 2002; accepted for publication 22 November 2002

\begin{abstract}
Summary. Women have lower iron stores than men because of iron loss during their reproductive years. However, variation between women could result from differences in iron loss, aspects of iron homeostasis common to men and women, or a combination of both. We compared the effects of age, menopause, menstrual blood loss and the number of pregnancies (sex-specific factors), and the effects of genetic variation, on markers of iron stores. We assessed how much the same genes or other familial factors influence iron status in both men and women. Data from 2039 female twins who participated in studies of reproductive health and iron status were used to estimate the proportions of variation that could be ascribed to genes, environment and measured factors. Significant effects of age, menopausal status and magnitude of menstrual blood loss were demonstrated, accounting for up to $18 \%$ of variance in serum
\end{abstract}

ferritin in this sample, but number of children had no significant effect. Genetic effects were more than twice as great as sex-specific effects. The within-pair similarity of ferritin values in dizygotic female twin pairs was greater than for dizygotic opposite-sex pairs, but this difference was not quite significant, consistent with a minor role for sex-specific factors; and the opposite-sex within-pair differences did not diminish significantly with age. We conclude that the contribution of genetic differences between women to variation in iron stores outweighs the comparatively small effects of interindividual variation in iron loss through variation in menstruation and number of pregnancies.

Keywords: ferritin, iron, menopause, menstruation, twin study.
Iron is an essential element and, although deficiency leads to anaemia, iron overload is also associated with a range of health problems. Therefore, iron stores are under substantial physiological control. We have shown previously that, in the population as a whole, iron status is subject to effects of variation in the HFE gene and also in other unidentified genes (Whitfield et al, 2000). However, sex-specific effects must also be considered; women have lower iron stores and are more vulnerable to iron deficiency than men. This difference is ascribed to their regular iron loss through menstrual bleeding and to the intermittent transfer of iron to the developing fetus during the reproductive years (see Baynes, 1994). After menopause, these sex-specific factors no longer operate, and iron stores increase. As iron absorption is regulated through a feedback loop involving body iron stores (see Skikne \& Baynes, 1994), the lower iron stores in premenopausal women are associated with greater iron absorption, bringing loss and absorption into balance.

Correspondence: John B. Whitfield, Department of Clinical Biochemistry, Royal Prince Alfred Hospital, Camperdown, NSW 2050, Australia. E-mail: John.Whitfield@email.cs.nsw.gov.au
Even among women, there are differences in the amount of menstrual (Hallberg et al, 1966; Rybo \& Hallberg, 1966; Hefnawi et al, 1980a,b; Hallberg \& Rossander-Hulten, 1991) and pregnancy-related iron loss. Such individual differences will lead to some women being more vulnerable than others to iron deficiency, especially if dietary iron supply is marginal or if closely spaced pregnancies impose an extra requirement for iron. There is evidence that menstrual variation results, in part, from genetic factors (Rybo \& Hallberg, 1966; Treloar et al, 1998). The nature of these genetic factors is unknown, but they could relate to differences in hormone levels, in uterine sensitivity to hormonal changes or (in relation to blood loss) coagulation and fibrinolysis mechanisms.

In addition, as alluded to above, variation in iron stores in women may result from factors that also influence iron stores in men. These would include not only variation within the HFE gene, which has been shown to affect the probability of iron deficiency in women (Beutler et al, 2000), but other genes, and possibly environmental factors, which might affect the setting or sensitivity of the iron stores/iron absorption feedback mechanism. If this is the 
most important mechanism, then the genetic factors that influence within-sex variation in iron stores will be common to both women and men and, to some extent, shared by opposite-sex siblings or dizygotic twins. If on the other hand, female-specific factors are of the greatest importance, then menstrual history, number of pregnancies and (where relevant) time since the menopause will be decisive, and women will be less similar to their brothers than to their sisters.

We have tested the relative importance of these two groups of factors using data from female twins and from male twins from dizygotic opposite-sex pairs. The women completed questionnaires on their obstetric and gynaecological history, and iron stores were assessed by blood tests. The aims of this paper are to compare the relative importance of variation in menstruation and fertility, and also the HFE C282Y and H63D genotypes and other genes affecting iron stores, in relation to serum iron, transferrin, transferrin saturation and ferritin concentrations. This allows us to infer the nature of the major factors influencing vulnerability to low iron stores and potential iron deficiency in women.

\section{MATERIALS AND METHODS}

\section{Subjects}

Subjects were recruited through the Australian Twin Registry. The original cohort of 4870 female twins and 2746 male twins was recruited between 1980 and 1982 (then aged 17-88 years), They participated in questionnaire-based studies on alcohol use, alcohol dependence, personality and lifestyle between 1980 and 1996, with blood samples being collected in 1993-96. Data from all female twins, and from male twins from female-male twin pairs, were used in this study.

The 1981 questionnaire survey. In 1980-82, as part of a health survey by mailed questionnaire, information was obtained from 1231 monozygotic female (MZF) and 748 dizygotic female (DZF) twin pairs (1979 female pairs) in a total sample of 3808 adult (i.e. over 18 years) twin pairs from the Australian National Health and Medical Research Council Twin Register ('1981 survey') (Jardine et al, 1984; Martin \& Jardine, 1986; Eaves et al, 1989). All twins were asked questions relating to personality, alcohol consumption and, for women, their menstrual and reproductive history. Three months before this survey, a pilot questionnaire was sent to a subsample of 100 twin pairs. Replies to both the pilot and the main questionnaire were received from 67 female twins, and these have been used to assess short-term reproducibility.

Two items concerning the twins' similarity in appearance and experiences of being mistaken by others were included to determine zygosity; pairs giving inconsistent responses were recontacted for clarification. Such questionnaires have been shown to give at least $95 \%$ agreement with diagnosis based on extensive blood typing (Martin \& Martin, 1975; Ooki et al, 1990). More recently, members of a subsample of 198 same-sex pairs from this group, who reported themselves to be monozygotic (MZ), were typed for 11 independent highly polymorphic markers in the course of an asthma study; no errors in our previous zygosity diagnosis were detected (Duffy, 1994; Treloar et al, 1999).

The 1989 questionnaire survey. The same twins were followed up 8 years later (Wave 2) between 1988 and 1990 when the minimum age of respondents was 25 years (Treloar et al, 1992; Heath et al, 1994, 1995). The 1989 questionnaire replicated most of the earlier questions and added new items on gynaecological and obstetric history (Treloar et al, 1998). In 1990, a small repeat survey was conducted to obtain 2-year test-retest reliability for information provided by respondents in Wave 2. The 1989 questionnaire was sent again to 500 female respondents, selected for being the first 500 to have returned the original questionnaire.

The gynaecological health study. This began in August 1993 (Treloar et al, 1999). Questionnaires were sent to members of 1570 female twin pairs and 158 individual female twins (3297 individuals) who responded to the 1989 (Wave 2) study. Individual twins in incomplete pairs were included for the purpose of validating hysterectomy and endometriosis, although their co-twins were no longer available for twin studies. The response rate was 94\% (3096 individuals).

Elderly patients' study. In 1993-95, a survey was commenced to study the health of twins aged over 50 years. The surveyed twins included a subset from Wave 2. This survey elicited information on ages at menarche, at menopause and (where appropriate) at hysterectomy. In order to clarify inconsistencies in reported menopausal age and complicating factors such as hysterectomy, hormone replacement therapy (HRT), pregnancy or breast-feeding, a small telephone study was conducted in 1996 on 146 female twins followed up to 1995.

Data collection

Relevant questions from the 1989 study are shown in Table I. Age at menopause was defined as age at the last

Table I. Questionnaire items from the 1989 survey.

1. How old were you when you had your first menstrual period?

2. Have you reached menopause ('natural change of life')

(a) How old were you when your periods stopped?

3. Have you had a hysterectomy?

(a) How old were you when you had your hysterectomy?

(b) Was the hysterectomy (1) Before (2) During or (3) After menopause?

(c) Were both ovaries removed?

If your periods are more or less regular, please answer this section

1. On average, how many days of bleeding do you have each time?

2. Are your periods?

$1=$ Heavy

2 = Moderate

$3=$ Light

Pregnancies

1. How many pregnancies have you had? Full term:/ Miscarriages:/Terminations: 
menstrual period, determined retrospectively, after a woman had stopped menstruating for 12 months not due to pregnancy, lactation or ill health. If a woman reported different ages at menopause in different surveys, we accepted the age at menopause reported in the earliest survey as the true one (assuming that the reported value was more reliable when the age of reporting was closer to the age at menopause) provided that there were no other inconsistencies in subsequent surveys. Some women reported natural menopause in an earlier survey but, in later surveys, reported that they were still menstruating. Such discrepancies were mainly related to HRT. If a woman reported an age at HRT that was older than the reported age at menopause, then she was classified as post-menopausal with age at menopause as first reported. If she reported receiving HRT treatment before menopause, or if no information on HRT or other reasons was given, then the subject was considered to be an unresolved case. If age at menopause was reported consistently in at least two surveys but the women reported that she was still menstruating at a later survey, we assumed that she had started HRT after natural menopause and she was classified as post-menopausal (Do et al, 1998). Years since menopause were calculated by subtracting age at menopause from age at blood collection. Age at menarche was taken from the earliest survey (Do et al, 1998).

Menstrual flow (heavy/moderate/light) and days of menstrual bleeding were taken from the 1989 survey, which was the most proximal to blood collection. The 3-month, 2-year and 8-year (Wave 1-2) repeatability coefficients ( \pm standard errors) were $0 \cdot 80 \pm 0 \cdot 11,0 \cdot 72 \pm 0 \cdot 07$ and $0 \cdot 48 \pm 0 \cdot 03$, respectively, suggesting strong shorter term and modest longer term stability of this measure (Treloar et al, 1998).

Body mass index (BMI) was computed using the formula: $\mathrm{BMI}=$ weight $(\mathrm{kg}) /[\text { height }(\mathrm{m})]^{2}$.

\section{Blood collection and analysis}

The subjects gave informed consent to blood collection and use of the blood. The studies were approved by both the Australian Twin Registry and the Bancroft Centre research ethics committee. The time of blood collection was recorded. Serum was separated and stored at $-70^{\circ} \mathrm{C}$ until analysed, and DNA was extracted from EDTA or heparin blood. Measurements of serum iron, transferrin and ferritin were performed using Roche methods on a Hitachi 917 analyser, and the percentage iron saturation of transferrin was calculated as $100 \times$ [iron] $/[$ transferrin $\times 25$ ] .

In all, 3375 subjects provided blood samples between 1993 and 1996. There were 3044 subjects with complete data, of whom 2039 were women. There were 352 female unpaired subjects (and 226 male), 724 pairs of femalefemale twins (481 monozygotic, 243 dizygotic) and 239 DZ opposite-sex (DZOS) pairs. There were also $181 \mathrm{MZ}$ male and $89 \mathrm{DZ}$ male twin pairs.

\section{Data analysis}

First, analysis of data from all the women was undertaken using the Mx 1.47 computer program (Neale, 1999). This program is designed for the analysis of data from twins and takes into account the sharing of genes within twin pairs, which might otherwise bias significance tests in a nonconservative direction. This first stage of analysis was conducted using the stepwise introduction of each of the independent variables, which allowed estimation of the significance of each variable entered and the proportion of the variance that it explained. This was done for each of the four markers of iron status, serum iron, transferrin, transferrin saturation and ferritin. Because of skewed distribution, ferritin values were log transformed before analysis. After the incorporation of measured variables (as shown in Table II) into the analysis, the magnitude of the additive genetic, shared environmental and non-shared environmental effects was estimated by fitting models of

Table II. The stepwise analysis of the factors affecting serum iron, transferrin, transferrin saturation and ferritin.

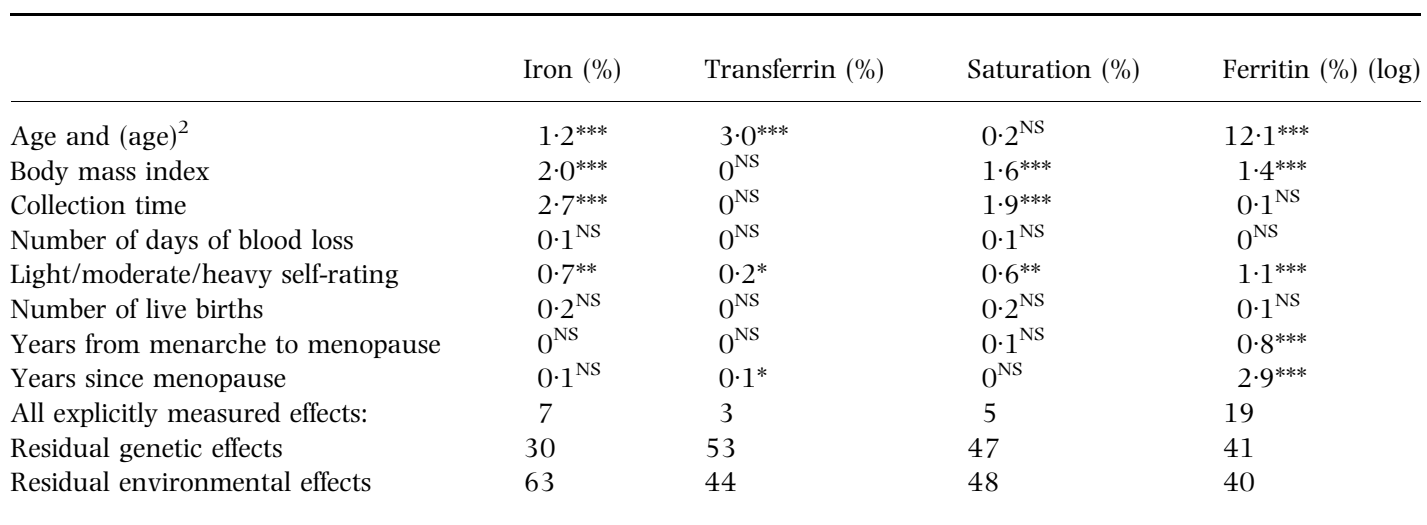

This analysis takes into account the effects of sharing of genes within twin pairs on the effective number of subjects. The variables listed were added to the model in the order shown so that the additional effects of each new variable are tested, using the improvement in goodness-of-fit of the model. The significance and the proportion of variance explained by each variable are shown.

${ }^{* * *} P<0.001 ;{ }^{* *} P<0.01 ;{ }^{*} P<0.05$; not significant (NS) $P>0.05$. 
sources of variation to the data for MZ and dizygotic (DZ) twin pairs.

Secondly, the variables that showed significant effects overall were examined in more detail. Attention was paid to the effects of menstrual blood loss, age and menopausal status. Subjects were categorized by their responses to relevant questionnaire items or assigned to 5-year groups for age and for time since menopause. Means and standard deviations were calculated for each group.

Because some factors potentially affecting iron status are exclusively female, whereas others are shared by both men and women, the similarity of the markers of iron status within dizygotic female-female and female-male pairs was compared. In view of the increase in ferritin values in women aged approximately 50 years and after menopause towards those found in men, we tested the hypothesis that female-specific effects on serum ferritin would decrease in importance with increasing age. This was done in two ways. First, we calculated within-pair correlation coefficients for all opposite-sex pairs and for all dizygotic same-sex female pairs, using multiple regression analysis and incorporating the effects of sex, age and (age $)^{2}$. Calculation of the correlations was repeated after dividing pairs into those aged under 50 years and those aged 50 years or more. Secondly, log-transformed ferritin results were z-transformed using the calculated means and standard deviations for all men, and for women by 5 -year age groups, and the within-DZ pair differences were calculated for the DZ female-female and the DZ female-male pairs. The mean squared within-pair differences for subjects under 50 years were compared against those for subjects aged 50 years or over using the F-ratio test.

\section{RESULTS}

Factors affecting iron stores

The results of the stepwise introduction of factors expected to influence markers of iron stores (serum iron, transferrin, transferrin saturation and ferritin) are shown in Table II. These results are restricted to data from women and, after adjustment for age and a quadratic term for age, BMI and collection time, the effects of variation in female-specific variables related to menstruation, pregnancies and menopausal status are shown. Finally, the residual effects of all genes (estimated from the comparison of MZ and DZ twin pair similarities) are included. Table II shows the proportion of the initial variance explained as each new independent variable is introduced, and the significance of the consequent improvement in fit between the model and the data. For the measured variables, the largest effect by far was that of age on serum ferritin. In addition, there were numerous significant effects accounting for $0 \cdot 5-3 \%$ of variance, but none of the dependent variables showed significant effects of the self-reported average days of menstrual blood loss or the number of live births. The estimated residual genetic effects were substantially greater than the effects of age or menopausal status, accounting for $47 \%$ of the variance in transferrin saturation and $41 \%$ of variance in log-transformed ferritin concentration.

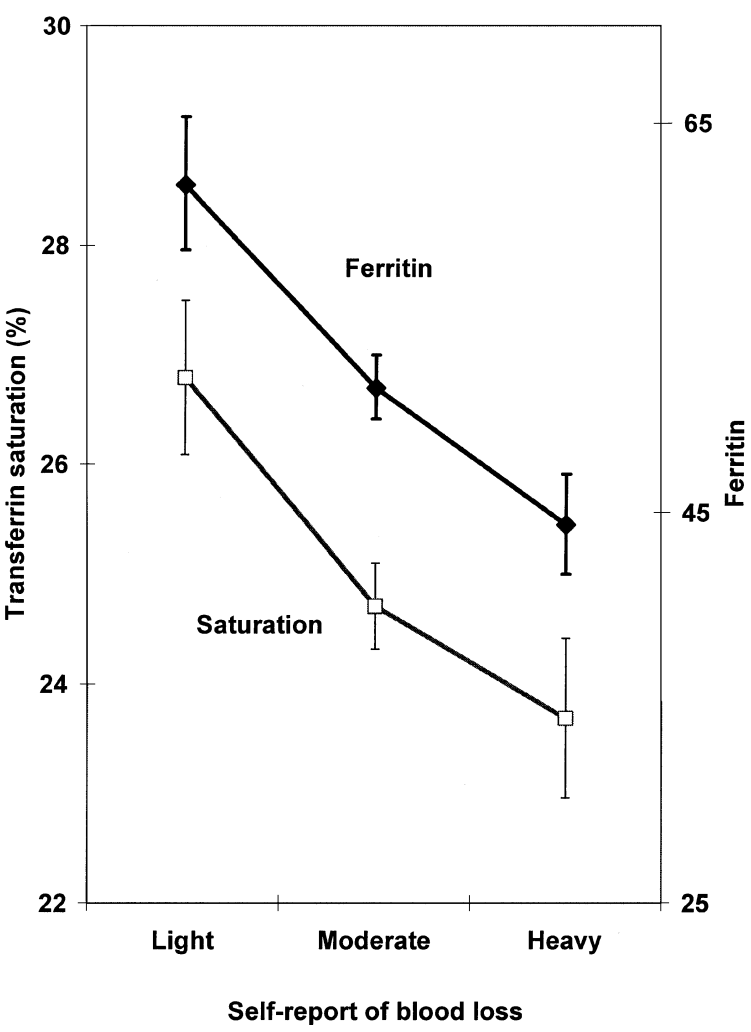

Fig 1. Effects of variation in self-reported menstrual blood loss on serum transferrin saturation and on serum ferritin. Error bars show standard errors of the means for each group, and the blood loss effects are significant $(P<0 \cdot 01)$ for both saturation and ferritin.

Factors affecting iron status in premenopausal women

There were no significant associations between iron storage markers and the number of pregnancies or the reported average duration of menstrual bleeding. However, the reported magnitude of blood loss (heavy/moderate/light) showed significant associations with subsequent measurements of ferritin, iron and saturation (see Table II). In each case, the order was light $>$ moderate $>$ heavy. The mean values for transferrin saturation and ferritin by blood loss category are illustrated in Fig 1.

\section{Age and menopausal status}

Data on menopausal status from the 1993 questionnaire were analysed in two ways. First, the effects of age alone (a highly significant factor in the stepwise analysis for women) were assessed by calculating the mean ferritin of 5-year age groups (Fig 2; data for men are shown for comparison). Secondly, pre- and post-menopausal women were compared; significant differences were found by this classification for ferritin, transferrin and iron, but not for saturation. Post-menopausal women were divided into groups according to the number of years since menopause. Only ferritin showed significant results by this classification, and values increased with increasing time since menopause. These additional effects of menopause, even after adjustment for age effects, are shown in Fig 3. 


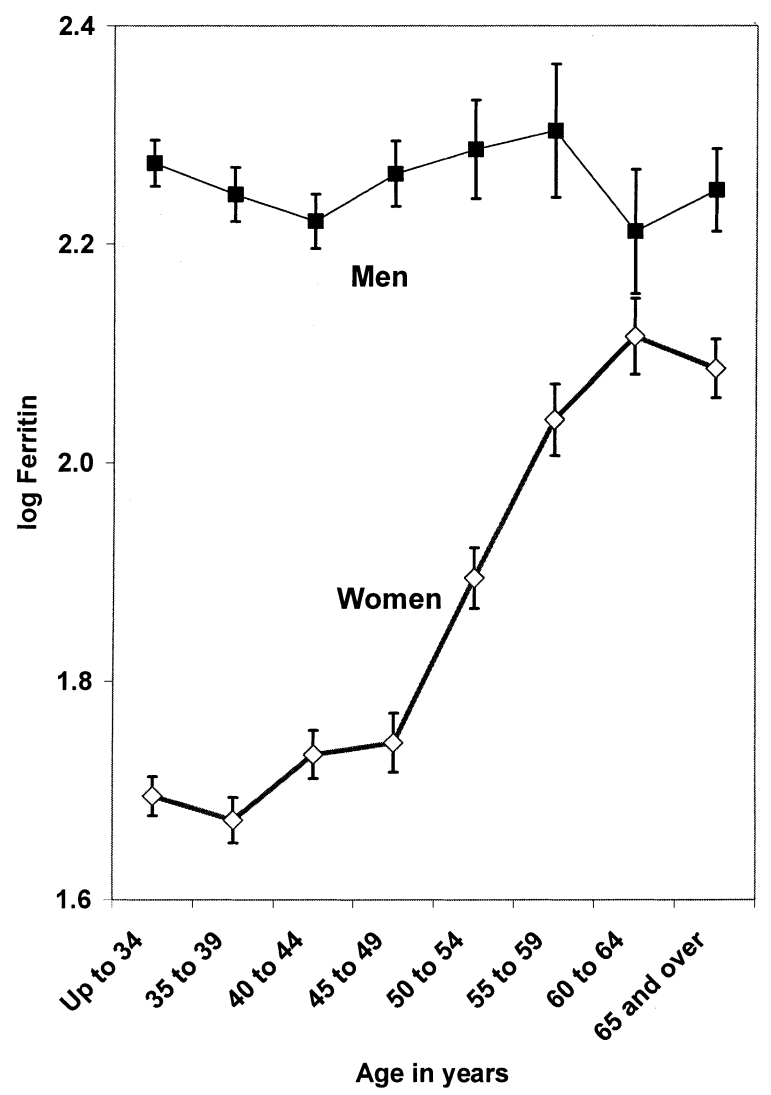

Fig 2. Change in mean serum ferritin with age in men and women. Error bars show standard errors of the means for each group.

Within-pair similarity in ferritin by age and sex

The within-pair correlation of log-transformed serum ferritin values, adjusted for the effects of sex, age and (age $)^{2}$, was less in opposite-sex pairs $(r=0 \cdot 18)$ than in same-sex female dizygotic pairs $(r=0 \cdot 30)$, but this difference was not significant $(P=0.082$, one-tail, with the expectation that the correlation would be greater in the same-sex pairs). Calculation of the pairwise correlations for DZF and DZOS twins was repeated after dividing subjects into those aged under 50 years and those aged 50 years or more, with results as shown in Table III. Although correlations in both age groups were higher in the same-sex pairs, the

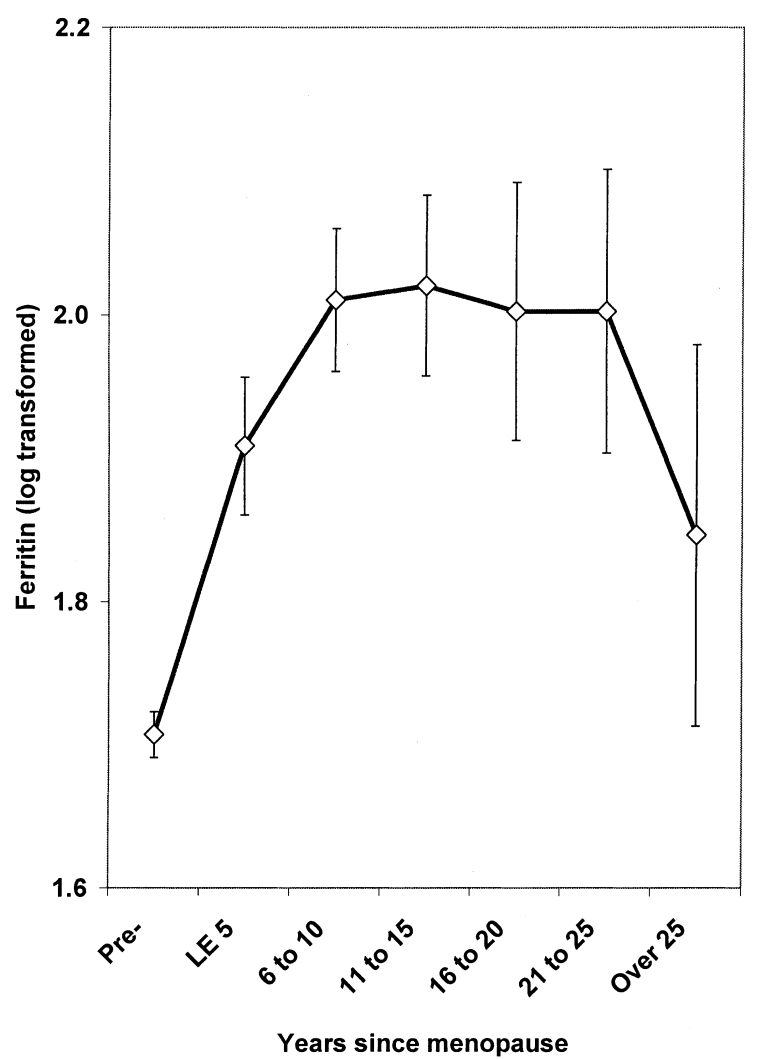

Fig 3. Effect of menopausal status and time since menopause on serum ferritin concentration. Error bars show standard errors of the means for each group.

differences were not significant with the number of pairs available. Moreover, the difference in sex- and age-adjusted within-pair mean squares between subjects aged below and above 50 years was just as great in the opposite-sex pairs as in the DZ female pairs (DZF: age under 50 years 1.50 versus age 50 years and over $1 \cdot 22$; DZOS: age under 50 years 1.55 versus age 50 years and over $1 \cdot 29$ ).

\section{DISCUSSION}

It is well established that women have lower iron stores than men and are consequently more susceptible to iron

Table III. The pairwise correlation coefficients for serum ferritin, comparing younger and older female-female (DZF) and female-male (DZOS) pairs.

\begin{tabular}{llll}
\hline & $\begin{array}{l}\text { DZF } \\
(243 \text { pairs })\end{array}$ & $\begin{array}{l}\text { DZOS } \\
(239 \text { pairs })\end{array}$ & $\begin{array}{l}P \text { (one-tail, } \\
\left.\mathrm{H}_{1}=\mathrm{DZF}>\mathrm{DZOS}\right)\end{array}$ \\
\hline All ages & $0 \cdot 30$ & $0 \cdot 18$ & $0 \cdot 082$ \\
Under 50 years only & $0 \cdot 26$ & $0 \cdot 15$ & $0 \cdot 154$ \\
50 years and over only & $0 \cdot 40$ & $0 \cdot 25$ & $0 \cdot 179$ \\
\hline
\end{tabular}

Correlations were calculated with adjustment for male-female differences in means, and for linear and quadratic terms for age in women. 
deficiency. The aim of this study was to compare the relative importance of female-specific factors, such as menstrual blood loss and pregnancies, and also non-sexspecific genetic factors, on the variation in markers of iron stores in women. From this, the relative importance of these two types of effects on the probability of iron deficiency can be inferred. In performing this analysis, we need to distinguish between factors that affect all women (compared with men) and factors that lead to differences between women.

The main marker of iron stores that can be used in studies of large numbers of presumably healthy subjects is serum ferritin. This has been validated against estimates of iron reserves through iron depletion (Skikne et al, 1990) and is widely used and, although it may be increased by inflammatory conditions, these would be uncommon in our subjects. Ferritin also increases with increasing alcohol consumption, but this was not significant among the women in this study (probably because high alcohol intake was rare). Techniques other than blood tests are either too invasive to be justified or require specialized equipment and the on-site attendance of all subjects. Measurement of serum iron and transferrin, and the calculation of transferrin saturation with iron, is useful in some situations, but these measures were not as responsive to known influences on iron status as ferritin in this study. Other tests, including serum transferrin receptor, are mainly useful in the differential diagnosis of anaemia rather than for assessment of iron stores.

As expected, age had a major effect on women's serum ferritin. Among our subjects, it accounted for $12 \%$ of the variation; the proportion is dependent on the actual age distribution of the participants and cannot be applied directly to populations with different age distributions, but it is clearly a major factor. However, this does not cause differences between women of the same age. Menopausal status and years since menopause had additional significant effects on serum ferritin, as shown in Table II and Fig 3. Ferritin increased above premenopausal values within 5 years of menopause, but a plateau in the ferritin concentration was not reached until 6-10 years. This is consistent with the age results shown in Fig 2, in which it can be seen that ferritin increased continuously between the 45-49 year and the 60-64 year age groups; this broader time span is presumably because of variation in age at menopause.

Information was obtained from the women regarding a number of variables that were expected to affect iron stores, such as number of children, years from menarche to menopause, average duration of monthly bleeding and selfassessed intensity of menstrual blood loss. Out of these, only the time from menarche to menopause and the intensity of menstrual blood loss showed significant effects. Both were comparatively minor sources of variation at $\approx 1 \%$ of the total variance. Intensity of menstrual blood loss affected serum iron and saturation as well as ferritin. The absence of any significant effect of number of children is surprising, but may be due to routine iron supplementation during pregnancy.
Turning to the non-sex-specific factors that could lead to differences in iron status between women, there was a significant but comparatively small effect of subjects' BMI. Ferritin increased, but iron and saturation decreased, with increasing BMI; the reason for this differential effect is not known, but an association between obesity and increased ferritin (but not increased transferrin saturation) in patients with unexplained iron overload has been reported (Moirand et al, 1997). Secondly, the variation in HFE genotype, either C282Y or H63D, is known to have significant effects on serum iron, transferrin and saturation, but the HFE effects on ferritin in women in this community-based sample account for $<2 \%$ of the variance (Whitfield et al, 2000). Other genes, which were not typed and the nature and location of which are still unknown, were found to have substantial effects on all markers; they represent the major source of differences in iron status and presumably therefore of the risk of iron deficiency between women.

Because iron stores increase after menopause, and because premenopausal women are subject to influences on iron status that do not apply to men, we postulated that the differences between brothers and sisters in the DZOS pairs would decrease after menopause. On one level, this is obviously true because mean values for women rise towards the male values after menopause. However, the relevant measure is the status of each woman or man in relation to others in that age and sex group, and so z-scores were calculated for each subject from the subgroup means and standard deviations. The within-pair differences in ferritin were then calculated for both DZF and DZOS pairs, and the mean squared differences for the younger and older groups were used to test whether siblings became more similar with age. Our expectation that results for opposite-sex pairs would converge after the expected age of menopause was only partially fulfilled: the ferritin of brothers and sisters did show a greater similarity, but the difference was not significant, and essentially the same degree of convergence was seen in the female same-sex pairs. Our interpretation of these results is that variation in menstruation and ages at menarche and menopause is of rather low heritability (Treloar et al, 1998) and that DZOS as well as DZF pairs show a greater similarity after the age of 50 years. This effect also accounts for the (non-significantly) higher within-pair correlations after age 50 years in both DZF and DZOS pairs, shown in Table III.

The interpretation of these results in the context of iron deficiency anaemia in women is subject to a number of caveats. The subjects were drawn from a volunteer twin registry broadly representative of the general community, not from patients presenting with possible anaemia. The prevalence of iron deficiency among our subjects was not high, and obviously depends on the criteria used and on age, but we have calculated previously (Whitfield et al, $2000)$ that serum ferritin values below $20 \mu \mathrm{g} / \mathrm{l}$ would be found in approximately one-fifth of women aged 40 years, excluding those homozygous for the HFE C282Y polymorphism. A transferrin saturation below 15\% would also be expected in $18 \%$ of homozygous HFE wild-type women at that age. These estimations did not include the effects of 
variation in menstrual blood loss, duration of reproductive life or years since menopause. However, such effects, although significant, were small when compared with the genetic effects, and we conclude that differences between women in their risk of low iron reserves is more influenced by genetic variation common to both sexes than by femalespecific obstetric or gynaecological factors. The nature of the genes affecting iron status in the general population remains unknown.

\section{ACKNOWLEDGMENTS}

Supported in part by grants AA07535 and AA07728 from the National Institute for Alcohol Abuse and Alcoholism, and by grants from the Australian National Health and Medical Research Council (941177 and 971232).

\section{REFERENCES}

Baynes, R.D. (1994) Iron deficiency. In: Iron Metabolism in Health and Disease (ed. by J.H. Brock, J.W. Halliday, M.J. Pippard \& L.W. Powell), pp. 189-225. W.B. Saunders, London.

Beutler, E., Felitti, V., Gelbart, T. \& Ho, N. (2000) The effect of HFE genotypes on measurements of iron overload in patients attending a health appraisal clinic. Annals of Internal Medicine, 133, 329-337.

Do, K.-A., Treloar, S.A., Pandeya, N., Purdie, D., Green, A.C., Heath, A.C. \& Martin, N.G. (1998) Predictive factors of age at menopause in a large Australian twin study. Human Biology, 70, 1073-1091.

Duffy, D.L. (1994) Asthma and Allergic Diseases in Australian Twins and their Families. PhD Thesis. University of Queensland, Brisbane, Australia.

Eaves, L.J., Eysenck, H.J. \& Martin, N.G. (1989) Genes, Culture and Personality: an Empirical Approach. Academic Press, London.

Hallberg, L. \& Rossander-Hulten, L. (1991) Iron requirements in menstruating women. American Journal of Clinical Nutrition, 54, 1047-1058.

Hallberg, L., Hogdahl, A.-M., Nilsson, L. \& Rybo, G. (1966) Menstrual blood loss - a population study. Variation at different ages and attempts to define normality. Acta Obstetricia et Gynecologica Scandinavica, 45, 320-351.

Heath, A.C., Bucholz, K.K., Slutske, W.S., Madden, P.A.F., Dinwiddie, S.H., Dunne, M.P., Statham, D.B., Whitfield, J.B., Martin, N.G. \& Eaves, L.J. (1994) The assessment of alcoholism in surveys in the general community: what are we measuring? Some insights from the Australian twin panel interview survey. International Review of Psychiatry, 6, 295-307.

Heath, A.C., Madden, P.A.F., Slutske, W.S. \& Martin, N.G. (1995) Personality and the inheritance of smoking behavior: a genetic perspective. Behavior Genetics, 25, 103-117.
Hefnawi, F., El-Zayat, A.F. \& Yacout, M.M. (1980a) Physiologic studies of menstrual blood loss. I. Range and consistency of menstrual blood loss in and iron requirements of menstruating Egyptian women. International Journal of Gynaecology and Obstetrics, 17, 343-348.

Hefnawi, F., el-Zayat, A.F. \& Yacout, M.M. (1980b) Physiologic studies of menstrual blood loss. II. Physiologic variables affecting the magnitude of menstrual blood loss. International Journal of Gynaecology and Obstetrics, 17, 348-352.

Jardine, R., Martin, N.G. \& Henderson, A.S. (1984) Genetic covariation between neuroticism and the symptoms of anxiety and depression. Genetic Epidemiology, 1, 89-107.

Martin, N.G. \& Jardine, R. (1986) Eysenck's contribution to behaviour genetics. In: Hans Eysenck: Consensus and Controversy (ed. by S. Modgil \& C. Modgil), pp. 13-47. Falmer Press, London.

Martin, N.G. \& Martin, P.G. (1975) The inheritance of scholastic abilities in a sample of twins. I. Ascertainment of the sample and diagnosis of zygosity. Annals of Human Genetics, 39, 213-218.

Moirand, R., Mortaji, A.M., Loreal, O., Paillard, F., Brissot, P. \& Deugnier, Y. (1997) A new syndrome of liver iron overload with normal transferrin saturation. Lancet, 349, 95-97.

Neale, M.C. (1999) Mx: Statistical Modeling, 5th edn. Department of Psychiatry, Richmond, VA.

Ooki, S., Yamada, K., Asada, A. \& Hayakawa, K. (1990) Zygosity diagnosis of twins by questionnaire. Acta Genetica et Medica Gemellologia (Roma), 39, 109-115.

Rybo, G. \& Hallberg, L. (1966) Influence of heredity and environment on normal menstrual blood loss. Acta Obstetricia et Gynecologica Scandinavica, 45, 389-410.

Skikne, B.S., Flowers, C.H. \& Cook, J.D. (1990) Serum transferrin receptor: a quantitative measure of tissue iron deficiency. Blood, 75, 1870-1876.

Skikne, B. \& Baynes, R.D. (1994) Iron absorption. In: Iron Metabolism in Health and Disease (ed. by J.H. Brock, J.W. Halliday, M.J. Pippard \& L.W. Powell), pp. 151-187. W.B. Saunders, London.

Treloar, S.A., Martin, N.G., Dennerstein, L., Raphael, B. \& Heath, A.C. (1992) Pathways to hysterectomy: insights from longitudinal twin research. American Journal of Obstetrics and Gynecology, 167, 82-88.

Treloar, S.A., Martin, N.G. \& Heath, A.C. (1998) Longitudinal genetic analysis of menstrual flow, pain, and limitation in a sample of Australian twins. Behavior Genetics, 28, 107-116.

Treloar, S.A., O'Connor, D.T., O'Connor, V.M. \& Martin, N.G. (1999) Genetic influences on endometriosis in an Australian twin sample. Fertility and Sterility, 71, 701-710.

Whitfield, J.B., Cullen, L., Powell, L.W., Heath, A.C., Zhu, G., Duffy, D.L. \& Martin, N.G. (2000) Effects of HFE C282Y and H63D polymorphisms, and polygenic background, on iron stores in a large community sample of twins. American Journal of Human Genetics, 66, 1246-1258. 